\title{
Études de lettres
}

de Lettres

3-4 | 2015

Représenter la corruption à l'âge baroque (1580-1660)

\section{Avant-propos}

\section{Frank Lestringant et Adrien Paschoud}

\section{OpenEdition}

\section{Journals}

Édition électronique

URL : http://journals.openedition.org/edl/897

DOI : $10.4000 /$ edl 897

ISSN : 2296-5084

\section{Éditeur}

Université de Lausanne

\section{Édition imprimée}

Date de publication : 15 décembre 2015

ISBN : 978-2-940331-47-5

ISSN : 0014-2026

\section{Référence électronique}

Frank Lestringant et Adrien Paschoud, « Avant-propos », Études de lettres [En ligne], 3-4 | 2015, mis en ligne le 01 décembre 2018, consulté le 20 décembre 2020. URL : http://journals.openedition.org/edl/ 897 ; DOI : https://doi.org/10.4000/edl.897 


\section{AVANT-PROPOS}

Douloureusement marquée par les guerres de Religion, la culture de la fin $\mathrm{du} \mathrm{XVI}^{\mathrm{e}}$ et du premier XVII ${ }^{\mathrm{e}}$ siècle, celle que l'on a naguère qualifiée de "baroque ${ }^{1}$, est saturée de références à l'état de corruption. Ouvrages de controverse confessionnelle, pamphlets hostiles à la figure royale, hagiographies et martyrologes, tragédies néo-sénéquiennes, théâtre d'édification, poésie de dévotion, maniérisme macabre, histoires tragiques, écrits démonologiques, traités médicaux, historiographie, natures mortes et vanités... autant de productions textuelles ou iconographiques destinées à rappeler à l'individu - du plus vil au plus noble - sa nature viciée, ou, du moins, l'inéluctable altération à la fois physique et morale qui l'accaparera. L'étymologie du mot "corruption" le montre assez: le suffixe cum-, sur lequel le mot corruptio est construit (corrumpere: "anéantir, briser, altérer») traduit l'intensité de la perte; il suggère un rapport différentiel au monde sensible et à l'expérience commune. En raison de son caractère rétrospectif et prospectif, la notion de corruption déploie un espace réfléchissant au sein duquel l'homme, la nature et l'univers sont envisagés dans leur dimension métamorphique, faisant ainsi se conjoindre passé, présent et futur, selon un rapport de causalité. Au tournant du $\mathrm{XVI}^{\mathrm{e}}$ et $\mathrm{du} \mathrm{XVII}^{\mathrm{e}}$ siècle, cette involution des êtres et des choses demeure soumise à deux interprétations diamétralement opposées, l'une qui lie toute forme de corruption à un cadre surplombant, en d'autres termes à une métaphysique qui la détermine en amont; l'autre qui tendra à naturaliser la notion, prenant alors le parti de refuser, ou du moins de contester toute posture abstraite de vérité.

I. Voir J. Rousset, La littérature de l'âge baroque, ainsi que Dernier regard sur le baroque dans lequel sont évoquées les difficultés définitoires inhérentes au "baroque» littéraire. Voir également D. Souiller (éd.), Le baroque en question(s). 
La notion de corruption doit d'abord et avant tout se lire à l'aune de l'anthropologie de la Chute et de la rédemption. Si elle assigne à l'homme une existence à la fois historique (elle lie tout individu au péché originel) et sotériologique (elle fonde la nécessité de gagner le salut), la culture chrétienne connaît cependant de nettes inflexions selon la conception de la grâce et du libre-arbitre qui lui est attachée. En dépit de fortes divergences doctrinales, les milieux réformés demeurent généralement fidèles à une interprétation rigoriste de la pensée augustinienne. Le sujet y est décrit comme un être pétri d'orgueil, à qui il est refusé de coopérer à son salut. L'humanité ne forme de fait qu'une massa perditionis, selon les termes de l'évêque d'Hippone. Sans doute, et même si d'importantes nuances s'imposent, la culture post-tridentine, dont la Compagnie de Jésus représente le fer de lance, se veut-elle plus accueillante: le péché originel n'a pas entièrement perverti l'individu. S'il y a prédestination, il ne s'agit que d'une prédestination au salut étant donné qu'elle nécessite pleinement l'adhésion du sujet via le corps ecclésiastique. Créé à l'image de Dieu, l'homme ne peut certes susciter le don de la grâce (l'initium fidei n'émane que de Dieu), mais il porte en lui les vestiges, au sens patristique du terme, de sa condition originelle ${ }^{2}$. Car l'expression de la corruption porte les traces d'une conscience anamorphique: l'absence de Dieu est précisément le gage de sa présence; c'est précisément parce que le monde est fallacieux qu'il invite l'homme à découvrir la Vérité. Tel est le principe qui traverse la littérature et l'iconographie des vanités ${ }^{3}$ et qui fait de la vacuité des représentations terrestres un opérateur de croyance, assurant ainsi la transition entre le visible et l'invisible. Sans cet art de la transition, mieux de la suture, entre nature et surnature, il n'est point de transcendance salvatrice. Plus encore, l'anthropologie de la Chute ne se lit qu'à la lumière d'une origine perdue, celle que seules la prédication, la méditation et la contemplation sont à même de recouvrer partiellement. La réflexion théologique, bien que mâtinée d'augustinisme, se réclame alors de la vertu théologale de l'espérance. Elle est tendue vers la promesse d'une vie exempte de tout déclin au sein de laquelle le martyr forme l'une des

2. Dans son Traité de l'amour de Dieu (1616), et plus particulièrement dans la partie IV, intitulée "De la décadence et ruine de la charité», François de Sales ne dira pas autre chose (Euvres, IV, I).

3. Voir J.-Ch. Darmon (dir.), Littérature et vanité, ainsi que le volume édité par A.-E. Spica, Discours et enjeux de la vanité. Voir également P. Rouillard, Les vanités dans la peinture du XVII siècle. 
figures centrales, réalisant pleinement la promesse par l'exemplarité qu'il offre aux hommes ${ }^{4}$.

En marge des grands modèles chrétiens - réformés ou post-tridentins - et de leur inscription dans la littérature et la pensée philosophique du temps ${ }^{5}$, émerge cependant une domestication de la notion. Si elle n'est pas niée, la pensée de la corruption se voit naturalisée, renouant ainsi avec la tradition antique de la phtora: la maladie est affranchie d'une lecture métaphorique pour ouvrir à une technè, une science des symptômes qui repose en grande partie sur la théorie humorale, du moins jusqu'au basculement épistémique induit par les travaux de William Harvey; de même, l'historiographie tend à soustraire les guerres civiles à une lecture exclusivement eschatologique ${ }^{6}$; les événements sont alors davantage soumis à une science des actions, à une herméneutique des arcana imperii dans la tradition tacitéenne ${ }^{7}$. A cet égard, et corrélativement, la pensée de Machiavel, considérée comme scandaleusement amorale, ouvre à une désacralisation du politique désormais inféodé au culte de la ruse et de la

4. La figure du martyr opère en cela un saut sacral dans la mesure où elle purifie par son geste le péché du monde. Voir F. Lestringant, "Témoignage et martyre».

5. L'accusation de corruption existe également au sein même des confessions réformée ou catholique. Il suffit de songer aux pamphlets anti-jésuites qui ont essaimé en France, sous l'impulsion du front gallican, et en Europe dès la fin du XVI ${ }^{\mathrm{e}}$ siècle, et dont le Catéchisme des jésuites (1602) d'Etienne Pasquier constitue l'archétype. Prônant une "dévotion aisée", les disciples de Loyola ne contredisent-ils pas les fondements même du message chrétien par une morale et une activités apostolique dévoyées? $\mathrm{Ne}$ sont-ils pas les maîtres de l'équivoque, rompant ainsi avec la simplicité chrétienne? $\mathrm{Ne}$ cherchent-ils pas à saper le pouvoir à partir de son centre, comme l'affirme l'auteur de l'Anticoton (1610)? Ne s'inspirent-ils pas en cela de la doctrine de Machiavel? (Voir P.-A. Fabre, C. Maire (éds), Les antijésuites). L’argument tacitéen de la "corruption de l'éloquence» est une manière de condamner l'asianisme des jésuites (voir M. Fumaroli, L'école du silence, p. 446-448).

6. Rapidement associés à un ensemble de métaphores mortifères, les troubles des Guerres de religion, de même que la hantise de la sédition (particulièrement aiguë après l'assassinat d'Henri IV), ont longtemps avivé une réflexion sur l'apogée et le déclin des grands empires dont le modèle paradigmatique remonte au moins à la République de Platon, via les écrits de Polybe. Il s'agit alors de penser les liens de consécution, de dérouler le fil des événements et de remonter aux origines afin de rendre intelligible les "fléaux» du temps présent. La marche de l'histoire humaine est soumise à des mouvements cycliques qui permettent de rendre compte de l'alternance entre stabilité politique et sédition, entre causes générales et causes particulières. Voir J.-M. Goulemot, $L e$ règne de l'histoire, en particulier p. 33-72.

7. Voir B. Guion, Du bon usage de l'histoire. 
prudence ${ }^{8}$. Cette rupture est en outre nourrie par l'imposante production littéraire relative au monde de la Cour et plus particulièrement à la figure du courtisan, celle que stigmatisera notamment Agrippa d'Aubigné dans Les Tragiques (1616). Prompt à revêtir divers masques, l'homme de cour offre alors une médiation nécessaire entre le pouvoir royal et l'ensemble du corps social mais, de fait, l'ontothéologie censée gouverner l'individu est brisée 9 . La notion de corruption touche également, par contraste, à une herméneutique renouvelée du sujet, dont le modèle paradigmatique se trouve dans les Essais de Montaigne, et plus largement dans le «connaistoi toi-même» socratique ${ }^{10}$. La corruption morale est écartée au profit d'un refus de toute norme absolue, de toute abstraction métaphysique; elle est interprétée à l'aune de la pensée sceptique qui essaime en France à partir de la fin du XVI e siècle ${ }^{11}$. S'il n'élude pas une forme de tragique, voire une conscience de la vanité de toute chose ${ }^{12}$, le libertinage érudit a formé, pour sa part, le lieu où s'énonce une parole singulière et singularisante, offrant en quelque sorte le pendant profane d'une foi intériorisée, moins portée au dolorisme ostentatoire ${ }^{13}$.

8. Les réformés, comme le clan ligueur, instrumentalisent la pensée de Machiavel dans des intentions purement polémiques, pour ce qui a trait notamment à la question du tyrannicide (voir A.-M. Battista, "Sur l'antimachiavélisme français du XVI $\mathrm{X}^{\mathrm{e}}$ siècle»). Sur la réception de Machiavel en France au XVII ${ }^{\mathrm{e}}$ siècle, voir E. Thuau, Raison d'Etat et pensée politique à l'époque de Richelieu. Richelieu fut souvent accusé par ses adversaires d'être un disciple du penseur florentin alliant tyrannie et immoralité, de même que Mazarin fut perçu comme un maître en fourberie.

9. Voir les travaux de J.-P. Cavaillé, notamment «De la construction des apparences au culte de la transparence».

Io. Dans ses Essais, Montaigne procède à une anatomie de lui-même, dont il admet qu'elle est guidée par une forme de perversité intrinsèque, affranchie toutefois d'une métaphysique (en cela Montaigne propose le modèle absolument inversé des Confessions de saint Augustin). Le sujet ne se pense plus en fonction d'un modèle vertical; il se pense par rapport à lui-même, dans la distance constitutive de sa conscience. Montaigne opère un glissement entre intention de se décrire et expression de soi, là où saint Augustin maintenait une frontière stricte entre l'esprit et la lettre (voir O. Pot, «De l'aliénation topique à la topique du sujet», en particulier p. 82-89).

II. Voir notamment P. F. Moreau (éd.), Le scepticisme au XVI et XVII e siècle.

I2. On aurait tort de limiter la production libertine - notamment poétique - à sa seule dimension charnelle; certaines œuvres témoignent, à l'image des sonnets de Des Barreaux, d'une conscience aiguë de la finitude de l'homme, tout en excluant une perspective sotériologique, car relevant de l'inconnaissable.

I3. Voir A. Gimaret, Extraordinaire et ordinaire des Croix, en particulier "Troisième partie». 
Réunissant littéraires, philosophes et historiens de la culture, ce volume se propose de visiter à nouveaux frais le double embranchement dans lequel la pensée de corruption se voit inscrite. Les contributions réunies ici ont pour dénominateur commun de ne pas considérer cette notion comme une sorte d'archétype ou un abandon aux puissances d'un imaginaire transhistorique. Il s'agit au contraire d'accorder une place de premier plan à la capacité des discours à représenter et à modéliser l'ancrage socio-historique et culturel dont ils sont issus et qu'ils réfractent différentiellement. La matière littéraire occupe à cet égard une position tout à fait particulière: elle circonscrit le monde dans un espace configurant; elle débrouille la diversité du réel, l'ordonne et, ce faisant, lui donne en retour une intelligibilité inédite. Dès lors, et à l'image du monstre qui produit aversion et étonnement ${ }^{14}$, l'expression de la corruption touche à un questionnement fondamental sur la mimèsis: sans doute davantage tributaire de l'héritage aristotélicien selon lequel la représentation des «choses horribles à regarder" (Poétique, V, 1448b) offre au lecteur une stylisation exemplaire et purificatrice des passions, elle n'en demeure pas moins marquée du sceau de la pensée platonicienne qui veut que le langage, séparé de sa transparence originelle, soit voué à l'échec dans sa tentative de restituer de la nature. La pensée de la corruption se situe sur une frontière esthétique instable. Parce qu'elle peint le dévoiement et l'abjection, elle va contre la «raison» et la "nature». Elle se rapproche en cela de la figuration $\mathrm{du}$ "grotesque» et des "chimères " de toutes espèces, telles que les avait stigmatisées toute une tradition, elle-même fondée sur les premières lignes de l'Art Poétique d'Horace et du célèbre Quidlibet audendi potestas accordé aux poètes comme aux peintres. Elle implique donc un écart entre le placere et le docere. Simultanément, toutefois, cet écart est pensé, problématisé, jamais occulté. La figuration de la corruption, comme celle de la monstruosité, jouit donc d'un statut tout à fait particulier : a) elle est exclue de l'art parce qu'elle est jugée indigne de toute représentation; b) elle est inclue dans le canon de la représentation en tant qu'instrument pathétique ou apologétique. Sans doute est-ce le propre de la littérature de dessiner des médiations symboliques, de reconstituer à partir du matériau informe des événements une cohérence. Dans le même temps, l'activité littéraire s'exerce dans une collectivité dans laquelle elle définit sa

I4. Outre les travaux de J. Céard, voir L. Daston et K. Park, Wonders and the Order of Nature (1150-1750), en particulier le chapitre V. 
sphère d'action; elle est plus généralement conditionné par un "horizon d'attente" au sens que l'esthétique de la réception a donné à ce terme, c'est-à-dire l'ensemble des conventions qui constituent et infléchissent les compétences du lectorat (considéré comme une entité collective) à une période donnée. Celle-ci est donc marquée par un ensemble complexe de stratégies (générique, thématique, poétique, intertextuelle) qui définissent son historicité.

Quelles que soient ses inflexions, la pensée de la corruption oscille selon ses acceptions entre un concept et une métaphore, d'où la polysémie d'une entité instrumentalisée à souhait. De fait, elle constitue un puissant outil heuristique en ce qu'elle dresse une véritable cartographie des maux présents. Drainant les savoirs et les discours, elle les fait dialoguer, les modélise, les confronte, les prolonge selon les circonstances, les lieux ou le sujet. Elle relève ainsi d'un cadre interprétatif d'une grande souplesse que ce volume collectif se propose d'arpenter à la lumière des diverses appropriations (théologique, politique, philosophique, historique, médicale, etc.) dont cette notion a été l'objet durant la période qui s'étend de la lente diffusion des principes post-tridentins, ponctuée de violentes controverses, à l'affirmation de l'absolutisme moderne ${ }^{15}$ qui tendra à inféoder le religieux au politique. C'est la raison pour laquelle les contributions réunies ici ont généralement soin de ne pas faire de la pensée de la corruption une entité stable, relayée sans heurts d'un auteur ou d'une ouvre à l'autre: cela reviendrait à céder à une illusion téléologique, héritière de l'histoire des idées. Le parcours proposé ici suggère au contraire que des paradigmes de représentation en apparence contradictoires peuvent coexister au sein d'une période historique, en s'opposant ou en s'ignorant. Inscrit dans une perspective transversale, ce volume entend faire apparaître les lignes de partage, mais aussi de rupture, qui traversent une entité soumise à des déterminations plurielles; aussi pourra-t-on mieux mesurer les procédures d'écriture qui président à une notion qui engage tour à tour une philosophie et une poétique, les deux niveaux étant le plus souvent indissociablement liés.

Frank Lestringant, Université de Paris-Sorbonne Adrien Paschoud, Université de Bâle

15. Il ne s'agira pas ici d'entrer dans les problématiques définitoires de ce terme. Pour une mise au point récente, voir F. Cosandey, R. Descimon, L'absolutisme en France. 


\section{BIBLIOGRAPHIE}

Battista, Anne-Marie, "Sur l'antimachiavélisme français du XVI siècle", Revue de synthèse, 130/3 (2009), p. 501-531.

Cavaillé, Jean-Pierre, «De la construction des apparences au culte de la transparence. Simulation et dissimulation entre le XVI ${ }^{\mathrm{e}}$ et le XVII ${ }^{\mathrm{e}}$ siècle", Littératures classiques, 34 (1998), p. 73-102.

Cosandey, Fanny, Descimon, Robert, L'absolutisme en France. Histoire et historiographie, Paris, Belin, 2002.

DARMON, Jean-Charles (dir.), Littérature et vanité: la trace de l'Ecclésiaste de Montaigne aux temps modernes, Paris, PUF, 2001.

Daston, Lorraine J., Park, Katharine, Wonders and the Order of Nature (1150-1750), New-York, Zone Book, 2001.

Fabre, Pierre-Antoine, Maire, Catherine (éds), Les antijésuites. Discours, figures et lieux de l'antijésuitisme à l'époque moderne, Rennes, Presses universitaires de Rennes, 2010.

François de Sales, Euvres, éd. par André Ravier, Paris, Gallimard, Bibliothèque de la Pléiade, 1969.

Fumaroli, Marc, L'école du silence. Le sentiment des images au XVII siècle, Paris, Flammarion, coll. Champs, 1998 (1994).

Gimaret, Antoinette, Extraordinaire et ordinaire des Croix. Les représentations du corps souffrant (1580-1650), Paris, Honoré Champion, 2011.

Goulemot, Jean-Marie, Le règne de l'histoire. Discours historiques et révolutions (XVII $-X V I I I^{\mathrm{e}}$ siècle), Paris, Albin Michel, 1996.

Guion, Béatrice, Du bon usage de l'histoire. Histoire, morale, politique à l'áge classique, Paris, Champion, 2008.

Lestringant, Franck, "Témoignage et martyre: donner à voir, donner à croire (XVI ${ }^{\mathrm{e}}$ - XVIII ${ }^{\mathrm{e}}$ siècle) ", Revue des Sciences Humaines, 269 (2003), p. 111-134.

Moreau, Pierre-François (éd.), Le scepticisme au XVI et XVII siècle, Paris, Albin Michel, 2001. 
Рот, Olivier, «De l'aliénation topique à la topique du sujet», in Emergence du sujet. De l' "Amant vert» au "Misanthrope», éd. par Olivier Pot, Genève, Droz, 2005, p. 49-113.

Rouillard, Philippe, Les vanités dans la peinture du XVII siècle: méditations sur la richesse, le dénuement et la rédemption, Paris, Albin Michel, 1990.

Rousset, Jean, La littérature de l'áge baroque, Paris, Corti, 1954.

—, Dernier regard sur le baroque, Paris, Corti, 1998.

Souiller, Didier (éd.), Le baroque en question(s), Paris, Honoré Champion, 1999 (Littératures classiques, 36).

Spica, Anne-Elisabeth (éd.), Discours et enjeux de la vanité, Paris, Armand Colin, 2005 (Littératures classiques, 56).

Thuau, Etienne, Raison d'Etat et pensée politique à l'époque de Richelieu, Paris, Albin Michel, 2000 (1966), p. 54-102. 